\title{
Lupaş post quantum Bernstein operators over arbitrary compact intervals
}

\author{
Khan A. ${ }^{1}$, Iliyas M. ${ }^{1}$, Mansoori M.S. ${ }^{1}$, Mursaleen M. ${ }^{1,2, 凶}$
}

This paper deals with Lupaş post quantum Bernstein operators over arbitrary closed and bounded interval constructed with the help of Lupaş post quantum Bernstein bases. Due to the property that these bases are scale invariant and translation invariant, the derived results on arbitrary intervals are important from computational point of view. Approximation properties of Lupaş post quantum Bernstein operators on arbitrary compact intervals based on Korovkin type theorem are studied. More general situation along all possible cases have been discussed favouring convergence of sequence of Lupaş post quantum Bernstein operators to any continuous function defined on compact interval. Rate of convergence by modulus of continuity and functions of Lipschitz class are computed. Graphical analysis has been presented with the help of MATLAB to demonstrate approximation of continuous functions by Lupaş post quantum Bernstein operators on different compact intervals.

Key words and phrases: post quantum calculus, post quantum Bernstein base, post quantum Bernstein operator, modulus of continuity, convergence criteria, rate of convergence.

\footnotetext{
${ }^{1}$ Department of Mathematics, Aligarh Muslim University, Aligarh 202002, India

${ }^{2}$ Department of Medical Research, China Medical University Hospital, China Medical University, Taichung, Taiwan

$\bowtie$ Corresponding author

E-mail: asifjnu07@gmail.com(Khan A.), iliyas2695@gmail.com (Iliyas M.),

shanawaz110592@gmail.com (Mansoori M.S.), mursaleenm@gmail.com (Mursaleen M.)
}

\section{Introduction}

In 1912, S.N. Bernstein [14] introduced the famous polynomials for any bounded function $f$ defined on $[0,1]$ as follows

$$
B_{\mu}(f ; x)=\sum_{r=0}^{\mu}\left(\begin{array}{c}
\mu \\
r
\end{array}\right) x^{r}(1-x)^{\mu-r} f\left(\frac{r}{\mu}\right), \quad x \in[0,1] .
$$

This sequence of operators $B_{\mu}: C[0,1] \rightarrow C[0,1]$ defined for any $\mu \in \mathbb{N}$ was proposed to provide a constructive proof of Weierstrass approximation theorem which attracted the attention of many researchers due to its applications in CAGD and other areas and is named as Bernstein polynomials [31].

In 1987, A. Lupaş [30] proposed the first $q$-analogue of Bernstein operators (rational) to approximate any continuous function defined on $[0,1]$ via $q$-calculus as follows

$$
L_{\mu, q}(f ; x)=\sum_{r=0}^{\mu} \frac{f\left(\frac{[r]_{q}}{[\mu]_{q}}\right)\left[\begin{array}{l}
\mu \\
r
\end{array}\right]_{q} q^{\frac{r(r-1)}{2}} x^{r}(1-x)^{\mu-r}}{\prod_{j=1}^{\mu}\left\{(1-x)+q^{j-1} x\right\}} .
$$

$\overline{\mathrm{y} \Delta \mathrm{K} 517.518 .8}$

2020 Mathematics Subject Classification:41A35, 41A36, 41A80.

(C) Khan A., Iliyas M., Mansoori M.S., Mursaleen M., 2021 
Ten years later, G.M. Phillips [42] introduced another generalization of the classical Bernstein operators (1) using $q$-calculus, the so-called Phillips $q$-Bernstein operators (polynomials) as follows

$$
B_{\mu, q}(f ; x)=\sum_{r=0}^{\mu}\left[\begin{array}{l}
\mu \\
r
\end{array}\right]_{q} x^{r} \prod_{s=0}^{\mu-r-1}\left(1-q^{s} x\right) f\left(\frac{[r]_{q}}{[\mu]_{q}}\right), \quad x \in[0,1],
$$

where $B_{\mu, q}: C[0,1] \rightarrow C[0,1]$ defined for any $\mu \in \mathbb{N}$ and any function $f \in C[0,1]$.

The Lupaş operators $L_{\mu, q}(f ; x)$ have an advantage of generating positive linear operators for all $q>0$, whereas Phillips polynomials generate positive linear operators for $0<q<1$.

Extension of quantum calculus ( $q$-calculus) is post quantum calculus $((p, q)$-calculus). Recently, first of all, M. Mursaleen et al. [35] applied concept of post quantum calculus or $(p, q)$-calculus in Approximation Theory and introduced $(p, q)$-analogue of Bernstein operators based on $(p, q)$-integers. They also introduced and studied several other operators based on post quantum calculus in [8-12,36-39]. For similar works in Approximation Theory and its applications in Computer Aided Geometric Design, one can refer to [1-7, 15, 17-19, 21-29, 32, 33, 41,44-48].

One of the advantage of using the extra parameter $p$ has been discussed in [39].

Firstly, we give certain notations and basic definitions from $(p, q)$-calculus. For any $p>0$ and $q>0$, the $(p, q)$ integers $[\mu]_{p, q}$ are defined by

$$
[\mu]_{p, q}=p^{\mu-1}+p^{\mu-2} q+p^{\mu-3} q^{2}+\cdots+p q^{\mu-2}+q^{\mu-1}= \begin{cases}\frac{p^{\mu}-q^{\mu}}{p-q}, & \text { when } p \neq q \neq 1, \\ \mu p^{\mu-1}, & \text { when } p=q \neq 1, \\ {[\mu]_{q},} & \text { when } p=1, \\ \mu, & \text { when } p=q=1,\end{cases}
$$

where $[\mu]_{q}$ denotes the $q$-integers and $\mu=0,1,2, \cdots$.

The formula for $(p, q)$-binomial expansion is as follows

$$
\begin{gathered}
(a x+b y)_{p, q}^{\mu}:=\sum_{r=0}^{\mu} p^{\frac{(\mu-r)(\mu-r-1)}{2}} q^{\frac{r(r-1)}{2}}\left[\begin{array}{c}
\mu \\
r
\end{array}\right]_{p, q} a^{\mu-r} b^{r} x^{\mu-r} y^{r}, \\
(x+y)_{p, q}^{\mu}=(x+y)(p x+q y)\left(p^{2} x+q^{2} y\right) \cdots\left(p^{\mu-1} x+q^{\mu-1} y\right), \\
(1-x)_{p, q}^{\mu}=(1-x)(p-q x)\left(p^{2}-q^{2} x\right) \cdots\left(p^{\mu-1}-q^{\mu-1} x\right),
\end{gathered}
$$

where $(p, q)$-binomial coefficients are defined by

$$
\left[\begin{array}{c}
\mu \\
r
\end{array}\right]_{p, q}=\frac{[\mu]_{p, q} !}{[r]_{p, q} ![\mu-r]_{p, q} !}
$$

For more details on $(p, q)$-calculus, one can refer to [17,35,39,43].

\section{Essential preliminaries and review of results}

M. Mursaleen et al. [35] introduced $(p, q)$-Bernstein operators for $0<q<p \leq 1$ to approximate any $f \in C[0,1]$ as follows

$$
B_{\mu, p, q}(f ; x)=\frac{1}{p^{\frac{\mu(\mu-1)}{2}}} \sum_{r=0}^{\mu}\left[\begin{array}{l}
\mu \\
r
\end{array}\right]_{p, q} p^{\frac{r(r-1)}{2}} x^{r} \prod_{s=0}^{\mu-r-1}\left(p^{s}-q^{s} x\right) f\left(\frac{[r]_{p, q}}{p^{r-\mu}[\mu]_{p, q}}\right), \quad x \in[0,1] .
$$


Note that the above operator approximate continuous function for sequence $q_{\mu}$ and $p_{\mu}$ satisfying $0<q_{\mu}<p_{\mu} \leq 1$.

Also when $p=1,(p, q)$-Bernstein operators given by (3) turns out to equation (2) which is known as Phillips $q$-Bernstein operators [42].

On the other hand, another sequence of operators as $(p, q)$-analogue of Lupaş Bernstein operators $L_{p, q}^{\mu}: C[0,1] \rightarrow C[0,1]$ is introduced by Kh. Khan, D.K. Lobiyal in [28], namely for all $p>0, q>0$,

$$
L_{p, q}^{\mu}(f ; x)=\sum_{k=0}^{\mu} \frac{f\left(\frac{p^{\mu-k}[k]_{p, q}}{[\mu]_{p, q}}\right)\left[\begin{array}{l}
\mu \\
k
\end{array}\right]_{p, q} p^{\frac{(\mu-k)(\mu-k-1)}{2}} q^{\frac{k(k-1)}{2}} x^{k}(1-x)^{\mu-k}}{\prod_{j=1}^{\mu}\left\{p^{j-1}(1-x)+q^{j-1} x\right\}} .
$$

Also note that in [28] authors have discussed only one case in which sequence of operators $L_{p, q}^{\mu}(f ; x)$ approximates continuous functions for sequence $q_{\mu}$ and $p_{\mu}$ satisfying $0<q_{\mu}<p_{\mu} \leq 1$. Now in this paper we reanalyse the convergence criteria and also discuss the other cases in which convergence hold by extending $[0,1]$ to $[\alpha, \beta]$.

The paper is organized as follows. In Section 2, we define a Lupaş $(p, q)$-analogue of Bernstein basis functions over $[\alpha, \beta]$ which will be used to construct the operator and which may be useful to construct Bézier curves and surfaces in CAGD. In Section 3, $(p, q)$-analogue of Lupaş operators are constructed and their approximation properties via Korovkin's type approximation theorem are presented and convergence criteria are reanalysed. The convergence criteria has been widely used by many mathematicians in their papers for different operators using quantum and post quantum calculus (see $[8,9,34]$ and [28, remark (5.1)]). In [16], Q.B. Cai and X.W. Xu indicated this as a basic problem of $(p, q)$-Bernstein-type operators (3), i.e. choosing $\left(p_{\mu}, q_{\mu}\right)$-integer satisfying $0<q_{\mu}<p_{\mu} \leq 1, \lim _{\mu \rightarrow \infty} p_{\mu}=1, \lim _{\mu \rightarrow \infty} q_{\mu}=1$ with $\lim _{\mu \rightarrow \infty}[\mu]_{p_{\mu}, q_{\mu}}=\infty$ for which the sequence of operators (3) converges uniformly to continuous functions defined on [0,1]. Kh. Khan, D.K. Lobiyal in [28] mentioned the following criteria for convergence in Remark (5.1): let $p_{\mu}$ and $q_{\mu}$ be the sequeces of real numbers satisfying $0<q_{\mu}<p_{\mu} \leq 1, \lim _{\mu \rightarrow \infty} p_{\mu}=1, \lim _{\mu \rightarrow \infty} q_{\mu}=1$ and $\lim _{\mu \rightarrow \infty} p_{\mu}^{\mu}=1$, $\lim _{\mu \rightarrow \infty} q_{\mu}^{\mu}=1$, then $\lim _{\mu \rightarrow \infty}[\mu]_{p_{\mu}, q_{\mu}}=\infty$ and $\lim _{\mu \rightarrow \infty} \frac{[\mu-1]_{p_{\mu}, q_{\mu}}}{[\mu]_{p_{\mu}, q_{\mu}}}=1$. Later in [8], author presented an assertion: let $q_{\mu}, p_{\mu}$ such that $0<q_{\mu}<p_{\mu} \leq 1$ and $q_{\mu} \rightarrow 1, p_{\mu} \rightarrow 1, q_{\mu} \rightarrow a, p_{\mu} \rightarrow b$ as $\mu \rightarrow \infty$, $a<b$, then $\lim _{\mu \rightarrow \infty}[\mu]_{p_{\mu}, q_{\mu}}=\infty$. In [16], authors did not give proof but they found out the class of sequences $p_{\mu}$ and $q_{\mu}$ satisfying the above criteria mentioned in [8]. We present an extended form of a convergence criteria by theorem and provide proofs. We state a theorem with proof which guarantees convergence. We show that when $p$ and $q$ are fixed numbers, then convergence can not be established in some cases. But convergence in those cases can also be achieved by choosing sequences $p_{\mu}$ and $q_{\mu}$ satisfying certain criteria.

Classical Bernstein basis function over arbitrary interval $[\alpha, \beta]$ is given by

$$
B_{r}^{\mu}(u ;[\alpha, \beta])=\left[\begin{array}{c}
\mu \\
r
\end{array}\right] \frac{(u-\alpha)^{r}(\beta-u)^{\mu-r}}{(\beta-\alpha)^{\mu}} \text { for } r=0,1, \ldots, \mu \text {. }
$$


P. Simeonova et al. defined quantum $q$-Bernstein basis functions for arbitrary interval [45] as follows

$$
B_{r}^{\mu}(u ;[\alpha, \beta] ; q)=\left[\begin{array}{c}
\mu \\
r
\end{array}\right]_{q} \frac{(u-\alpha) \ldots\left(u-q^{r-1} \alpha\right)(\beta-u) \ldots\left(\beta-q^{\mu-r-1} u\right)}{(\beta-\alpha) \ldots\left(\beta-q^{\mu-1} \alpha\right)} \quad \text { for } \quad r=0,1, \ldots, \mu \text {. }
$$

Motivated by the work of Kh. Khan, D.K. Lobiyal [28], we extend post quantum Bernstein basis functions and sequence of operators over arbitrary interval $[\alpha, \beta]$.

\section{Construction of post quantum Lupaş basis functions on interval $[\alpha, \beta]$}

Extension of Lupaş type post quantum analogue of the Bernstein functions (rational) over $[\alpha, \beta]$ using post quantum binomial expansion is defined as follows.

For any $p>0$ and $q>0$, we set

$$
b_{p, q}^{r, \mu}(t ; \alpha, \beta)=\frac{\left[\begin{array}{l}
\mu \\
r
\end{array}\right]_{p, q} p^{\frac{(\mu-r)(\mu-r-1)}{2}} q^{\frac{r(r-1)}{2}}(t-\alpha)^{r}(\beta-t)^{\mu-r}}{\prod_{j=1}^{\mu}\left\{p^{j-1}(\beta-t)+q^{j-1}(t-\alpha)\right\}},
$$

where $b_{p, q}^{0, \mu}(t ; \alpha, \beta), b_{p, q}^{1, \mu}(t ; \alpha, \beta), \cdots, b_{p, q}^{\mu, \mu}(t ; \alpha, \beta)$ are the $(p, q)$-analogue of the Lupas $q$-Bernstein functions [20] of degree $\mu$ on the interval $[\alpha, \beta]$.

Some observations about basis functions (5) are listed below.

1. When $p=1$ and $\alpha=0, \beta=1$, Lupaş post quantum Bernstein functions over $[\alpha, \beta]$ turns out to be Lupaş $q$-Bernstein functions as given in [20], whereas when $p=q=1$, and $\alpha=0, \beta=1$, Lupaş post quantum Bernstein functions turns out to be classical Bernstein functions.

2. These basis functions are translation invariant and scale invariant both. While basis of operator (3) are not translation invariant but scale invariant.

Translation invariance: for any real number $c$

$$
b_{p, q}^{r, \mu}(t+c ; \alpha+c, \beta+c)=b_{p, q}^{r, \mu}(t ; \alpha, \beta)
$$

Scale invariance: for any nonzero real number $c \neq 0$

$$
b_{p, q}^{r, \mu}(c t ; c \alpha, c \beta)=b_{p, q}^{r, \mu}(t ; \alpha, \beta) .
$$

3. These basis functions (5) forms the basis of operator (4) for the values $\alpha=0$ and $\beta=1$.

4. These basis functions are nonnegative for all $p, q>0$, while basis functions of operator (3) are nonnegative on interval $[0,1]$ for $0<q<p<\infty$.

Further, we construct sequence of operators over an arbitrary interval $[\alpha, \beta]$, using these basis functions (5) and derive some results which will be useful from computational aspects. 


\section{Construction of operators on arbitrary compact intervals}

Now, we present post quantum analogue of Lupaş Bernstein operators over $[\alpha, \beta]$ as follows.

For any $p>0$ and $q>0$, the operators $L_{p, q}^{\mu, \alpha, \beta}: C[\alpha, \beta] \rightarrow C[\alpha, \beta]$ are defined by

$$
L_{p, q}^{\mu, \alpha, \beta}(f ; x)=\sum_{r=0}^{\mu} \frac{f\left(\alpha+\frac{(\beta-\alpha) p^{\mu-r}[r]_{p, q}}{[\mu]_{p, q}}\right)\left[\begin{array}{l}
\mu \\
r
\end{array}\right]_{p, q} p^{\frac{(\mu-r)(\mu-r-1)}{2}} q^{\frac{r(r-1)}{2}}(x-\alpha)^{r}(\beta-x)^{\mu-r}}{\prod_{j=1}^{\mu}\left\{p^{j-1}(\beta-x)+q^{j-1}(x-\alpha)\right\}} .
$$

Again, when $p=1$, and $\alpha=0, \beta=1$, post quantum Lupaş Bernstein operators turns out to be Lupaş $q$-Bernstein operators as given in [30].

When $p=q=1, \alpha=0, \beta=1$ post quantum Lupaş Bernstein operators on $[\alpha, \beta]$ turns out to be classical Bernstein operators.

It follows directly from the definition that operators $L_{p, q}^{\mu, \alpha, \beta}(f ; t)$ possess the end point interpolation property, that is

$$
L_{p, q}^{\mu, \alpha, \beta}(f ; \alpha)=f(\alpha), \quad L_{p, q}^{\mu, \alpha, \beta}(f ; \beta)=f(\beta)
$$

for all $p>0$ and $q>0$, and all $\mu=1,2, \cdots$.

Clearly the sequence of operators $L_{p, q}^{\mu, \alpha, \beta}(t)$ is positive for all $p>0, q>0$. Also it is linear as $L_{p, q}^{\mu, \alpha, \beta}(a f+b g)=a L_{p, q}^{\mu, \alpha, \beta}(f)+b L_{p, q}^{\mu, \alpha, \beta}(g)$ for all $f, g \in C[\alpha, \beta]$ and $a, b \in \mathbb{R}$. Hence, $L_{p, q}^{\mu, \alpha, \beta}(t)$ is positive linear operator on $C[\alpha, \beta]$.

\section{Some auxillary results for Chebyshev test functions}

The following properties hold:

(1) $L_{p, q}^{\mu, \alpha, \beta}\left(1 ; \frac{\beta u+\alpha}{u+1}\right)=1$,

(2) $L_{p, q}^{\mu, \alpha, \beta}\left(t ; \frac{\beta u+\alpha}{u+1}\right)=\frac{\beta u+\alpha}{u+1}$,

(3) $L_{p, q}^{\mu, \alpha, \beta}\left(t^{2} ; \frac{\beta u+\alpha}{u+1}\right)=\alpha^{2}+\left(\frac{u}{u+1} \frac{p^{\mu-1}}{[\mu]_{p, q}}+\frac{q u}{u+1}\left(\frac{q u}{p+q u}\right) \frac{[\mu-1]_{p, q}}{[\mu]_{p, q}}\right)(\beta-\alpha)^{2}+2 \alpha(\beta-\alpha) \frac{u}{u+1}$,

or equivalently for $x=\frac{\beta u+\alpha}{u+1}$

(1) $L_{p, q}^{\mu, \alpha, \beta}(1 ; x)=1$,

(2) $L_{p, q}^{\mu, \alpha, \beta}(t ; x)=x$,

(3) $L_{p, q}^{\mu, \alpha, \beta}\left(t^{2} ; x\right)=\alpha^{2}+\left(\frac{p^{\mu-1}(x-\alpha)}{[\mu]_{p, q}(\beta-\alpha)}+\frac{q^{2}(x-\alpha)^{2}}{(\beta-\alpha)(p(\beta-x)+q(x-\alpha))} \frac{[\mu-1]_{p, q}}{[\mu]_{p, q}}\right)(\beta-\alpha)^{2}+2 \alpha(x-\alpha)$.

Proof. Using post quantum binomial expansion, (1) is obvious, namely

$$
L_{p, q}^{\mu, \alpha, \beta}\left(1 ; \frac{\beta u+\alpha}{u+1}\right)=\sum_{r=0}^{\mu} \frac{\left[\begin{array}{l}
\mu \\
r
\end{array}\right]_{p, q} p^{\frac{(\mu-r)(\mu-r-1)}{2}} q^{\frac{r(r-1)}{2}} u^{r}}{\prod_{j=1}^{\mu}\left\{p^{j-1}+q^{j-1} u\right\}}=1 .
$$


(2)

$$
\begin{aligned}
& L_{p, q}^{\mu, \alpha, \beta}\left(t ; \frac{\beta u+\alpha}{u+1}\right)=\sum_{r=0}^{\mu} \frac{\left(\alpha+(\beta-\alpha) \frac{\left.p^{\mu-r}[r]\right]_{p, q}}{[\mu]_{p, q}}\right)\left[\begin{array}{l}
\mu \\
r
\end{array}\right]_{p, q} p^{\frac{(\mu-r)(\mu-r-1)}{2}} q^{\frac{r(r-1)}{2}} u^{r}}{\prod_{j=1}^{\mu}\left\{p^{j-1}+q^{j-1} u\right\}} \\
& =\sum_{r=0}^{\mu} \frac{\left(\alpha+(\beta-\alpha) \frac{p^{\mu-r}[r]_{p, q}}{[\mu]_{p, q}}\right)\left[\begin{array}{l}
\mu \\
r
\end{array}\right]_{p, q} p^{\frac{(\mu-r)(\mu-r-1)}{2}} q^{\frac{r(r-1)}{2}} u^{r}}{\prod_{j=0}^{\mu-1}\left\{p^{j}+q^{j} u\right\}} \\
& L_{p, q}^{\mu, \alpha, \beta}\left(t ; \frac{\beta u+\alpha}{u+1}\right)=\alpha+\sum_{r=0}^{\mu} \frac{(\beta-\alpha) p^{\mu-r}\left[\begin{array}{c}
\mu-1 \\
r-1
\end{array}\right]_{p, q} p^{\frac{(\mu-r)(\mu-r-1)}{2} q^{\frac{r(r-1)}{2}} u^{r}}}{\prod_{j=0}^{\mu-1}\left\{p^{j}+q^{j} u\right\}} \\
& =\alpha+\sum_{r=1}^{\mu} \frac{(\beta-\alpha) p^{\mu-r}\left[\begin{array}{c}
\mu-1 \\
r-1
\end{array}\right]_{p, q} p^{\frac{(\mu-r)(\mu-r-1)}{2}} q^{\frac{r(r-1)}{2}} u^{r}}{\prod_{j=0}^{\mu-1}\left\{p^{j}+q^{j} u\right\}} \\
& \Rightarrow L_{p, q}^{\mu, \alpha, \beta}\left(t ; \frac{\beta u+\alpha}{u+1}\right)=\alpha+(\beta-\alpha) \sum_{r=0}^{\mu-1} \frac{p^{\mu-r-1}\left[\begin{array}{c}
\mu-1 \\
r
\end{array}\right]_{p, q} p^{\frac{(\mu-r-1)(\mu-r-2)}{2} q^{\frac{r(r+1)}{2}} u^{r+1}}}{\prod_{j=0}^{\mu-1}\left\{p^{j}+q^{j} \mathcal{u}\right\}} \\
& =\alpha+\frac{(\beta-\alpha) u}{u+1} \sum_{r=0}^{\mu-1} \frac{p^{\mu-1}\left[\begin{array}{c}
\mu-1 \\
r
\end{array}\right]_{p, q} p^{\frac{(\mu-r-1)(\mu-r-2)}{2}} q^{\frac{r(r-1)}{2}}\left(\frac{q u}{p}\right)^{r}}{\prod_{j=0}^{\mu-2}\left\{p^{j} p+q^{j}(q u)\right\}} \\
& \Rightarrow L_{p, q}^{\mu, \alpha, \beta}\left(t ; \frac{\beta u+\alpha}{u+1}\right)=\alpha+\frac{(\beta-\alpha) u}{u+1} \sum_{r=0}^{\mu-1} \frac{\left[\begin{array}{c}
\mu-1 \\
r
\end{array}\right]_{p, q} p^{\frac{(\mu-r-1)(\mu-r-2)}{2}} q^{\frac{r(r-1)}{2}}\left(\frac{q u}{p}\right)^{r}}{\prod_{j=0}^{\mu-2}\left\{p^{j}+q^{j}\left(\frac{q u}{p} u\right)\right\}} \\
& =\alpha+\frac{(\beta-\alpha) u}{u+1}=\frac{\beta u+\alpha}{u+1}
\end{aligned}
$$

or equivalently for $x=\frac{\beta u+\alpha}{u+1}$

$$
L_{p, q}^{\mu, \alpha, \beta}(t, x)=x
$$

(3) To prove, $L_{p, q}^{\mu, \alpha, \beta}\left(t^{2}, x\right)=\alpha^{2}+\left(\frac{p^{\mu-1}(x-\alpha)}{[\mu]_{p, q}(\beta-\alpha)}+\frac{q^{2}(x-\alpha)^{2}}{(\beta-\alpha)(p(\beta-x)+q(x-\alpha))} \frac{[\mu-1]_{p, q}}{[\mu]_{p, q}}\right)(\beta-\alpha)^{2}+$ $2 \alpha(x-\alpha)$, consider 


$$
\begin{aligned}
& L_{p, q}^{\mu, \alpha, \beta}\left(t^{2} ; \frac{\beta u+\alpha}{u+1}\right)=\sum_{r=0}^{\mu} \frac{\left(\alpha+(\beta-\alpha) \frac{p^{\mu-r}[r]_{p, q}}{[\mu]_{p, q}}\right)^{2}\left[\begin{array}{l}
\mu \\
r
\end{array}\right]_{p, q} p^{\frac{(\mu-r)(\mu-r-1)}{2}} q^{\frac{r(r-1)}{2}} u^{r}}{\prod_{j=0}^{\mu-1}\left\{p^{j}+q^{j} u\right\}} \\
& =(\beta-\alpha)^{2} \sum_{r=0}^{\mu} \frac{p^{2 \mu-2 r} \frac{[r]_{p, q}}{[\mu]_{p, q}}\left[\begin{array}{c}
\mu-1 \\
r-1
\end{array}\right]_{p, q} p^{\frac{(\mu-r)(\mu-r-1)}{2}} q^{\frac{r(r-1)}{2}} u^{r}}{\prod_{j=0}^{\mu-1}\left\{p^{j}+q^{j} u\right\}} \\
& +\alpha^{2}+2 \alpha(\beta-\alpha) \sum_{r=0}^{\mu} \frac{p^{\mu-r} \frac{[r]_{p, q}}{[\mu]_{p, q}}\left[\begin{array}{c}
\mu \\
r
\end{array}\right]_{p, q} p^{\frac{(\mu-r)(\mu-r-1)}{2}} q^{\frac{r(r-1)}{2}} u^{r}}{\prod_{j=0}^{\mu-1}\left\{p^{j}+q^{j} u\right\}} \\
& =(\beta-\alpha)^{2} \frac{u}{u+1} \sum_{r=0}^{\mu-1} p^{\mu-1-r} \frac{[r+1]_{p, q}}{[\mu]_{p, q}} \frac{\left[\begin{array}{c}
\mu-1 \\
r
\end{array}\right]_{p, q} p^{\frac{(\mu-r-1)(\mu-r-2)}{2}} q^{\frac{r(r-1)}{2}}\left(\frac{q u}{p}\right)^{r}}{\prod_{j=0}^{\mu-2}\left\{p^{j}+q^{j}\left(\frac{q}{p} u\right)\right\}} \\
& +\alpha^{2}+2 \alpha(\beta-\alpha) \frac{u}{u+1} \\
& \Rightarrow L_{p, q}^{\mu, \alpha, \beta}\left(t^{2} ; \frac{\beta u+\alpha}{u+1}\right)=(\beta-\alpha)^{2} \frac{u}{u+1} \frac{[\mu-1]_{p, q}}{[\mu]_{p, q}} \\
& \times \sum_{r=0}^{\mu-1} p^{\mu-1-r}\left(\frac{p^{r}+q[r]_{p, q}}{[\mu-1]_{p, q}}\right) \frac{\left[\begin{array}{c}
\mu-1 \\
r
\end{array}\right]_{p, q} p^{\frac{(\mu-r-1)(\mu-r-2)}{2}} q^{\frac{r(r-1)}{2}\left(\frac{q u}{p}\right)^{r}}}{\prod_{j=0}^{\mu-2}\left\{p^{j}+q^{j}\left(\frac{q}{p} u\right)\right\}} \\
& +\alpha^{2}+2 \alpha(\beta-\alpha) \frac{u}{u+1}=(\beta-\alpha)^{2}\left(\frac{u}{u+1} \frac{p^{\mu-1}}{[\mu]_{p, q}}\right. \\
& \left.+\frac{q u}{u+1} \frac{[\mu-1]_{p, q}}{[\mu]_{p, q}} \sum_{r=0}^{\mu-1} p^{n-r-1} \frac{\left[\begin{array}{c}
\mu-2 \\
r-1
\end{array}\right]_{p, q} p^{\frac{(\mu-r-1)(\mu-r-2)}{2} q^{\frac{r(r-1)}{2}}\left(\frac{q u}{p}\right)^{r}}}{\prod_{j=0}^{\mu-2}\left\{p^{j}+q^{j}\left(\frac{q}{p} u\right)\right\}}\right) \\
& +\alpha^{2}+2 \alpha(\beta-\alpha) \frac{u}{u+1} \\
& \Rightarrow L_{p, q}^{\mu, \alpha, \beta}\left(t^{2} ; \frac{\beta u+\alpha}{u+1}\right)=(\beta-\alpha)^{2}\left(\frac{u}{u+1} \frac{p^{\mu-1}}{[\mu]_{p, q}}+\frac{q u}{u+1} \frac{[\mu-1]_{p, q}}{[\mu]_{p, q}} \frac{\frac{q u}{p}}{1+\frac{q u}{p}}\right. \\
& \left.\times \sum_{r=0}^{\mu-2} p^{\mu-2} \frac{\left[\begin{array}{c}
\mu-2 \\
r
\end{array}\right]_{p, q} p^{\frac{(\mu-r-2)(\mu-r-3)}{2}} q^{\frac{r(r-1)}{2}\left(\frac{q^{2} u}{p^{2}}\right)^{r}}}{p^{\mu-2} \prod_{j=0}^{\mu-3}\left\{p^{j}+q^{j}\left(\frac{q^{2}}{p^{2}} u\right)\right\}}\right)+\alpha^{2}+2 \alpha(\beta-\alpha) \frac{u}{u+1} \\
& =\alpha^{2}+(\beta-\alpha)^{2}\left(\frac{u}{u+1} \frac{p^{\mu-1}}{[\mu]_{p, q}}+\frac{q u}{u+1}\left(\frac{q u}{p+q u}\right) \frac{[\mu-1]_{p, q}}{[\mu]_{p, q}}\right)+2 \alpha(\beta-\alpha) \frac{u}{u+1}
\end{aligned}
$$

or equivalently for $x=\frac{\beta u+\alpha}{u+1}$,

$L_{p, q}^{\mu, \alpha, \beta}\left(t^{2} ; x\right)=\alpha^{2}+\left(\frac{p^{\mu-1}(x-\alpha)}{[\mu]_{p, q}(\beta-\alpha)}+\frac{q^{2}(x-\alpha)^{2}}{(\beta-\alpha)(p(\beta-x)+q(x-\alpha))} \frac{[\mu-1]_{p, q}}{[\mu]_{p, q}}\right)(\beta-\alpha)^{2}+2 \alpha(x-\alpha)$. 
Remark 1. Clearly that $L_{p, q}^{\mu, \alpha, \beta}(A t+B)=A x+B$ for all $p>0$ and $q>0, A, B \in \mathbb{R}$ and for all $\mu=1,2,3, \cdots$. It means that operator $L_{p, q}^{\mu, \alpha, \beta}(t)$ preserves linear test functions.

\section{Convergence criteria}

Now, we analyse the conditions for convergence by sequence of operators (6) to any continuous function defined on $[\alpha, \beta]$.

Remark 2. For $q>0$ and $p>0$, it is easy to compute that $\lim _{\mu \rightarrow \infty}[\mu]_{p, q}=0$ or $\frac{1}{p-q}$ or $\frac{1}{q-p}$ or $\infty$ and $\lim _{\mu \rightarrow \infty} \frac{[\mu-1]_{p, q}}{[\mu]_{p, q}}=1$ or $\frac{1}{p}$ or $\frac{1}{q}$.

Consider the following cases.

Case 1. When $0<q<p<1$, then $\lim _{\mu \rightarrow \infty}[\mu]_{p, q}=0$ and $\lim _{\mu \rightarrow \infty} \frac{[\mu-1]_{p, q}}{[\mu]_{p, q}}=\frac{1}{p}$.

Proof. Recall that $[\mu]_{p, q}=\frac{p^{\mu}-q^{\mu}}{p-q}$, as both $p$ and $q$ satisfies $0<p<1,0<q<1$. So $\lim _{\mu \rightarrow \infty} p^{\mu}=0$ and $\lim _{\mu \rightarrow \infty} q^{\mu}=0$, hence $\lim _{\mu \rightarrow \infty} \frac{p^{\mu}-q^{\mu}}{p-q}=0$. Now, $[\mu-1]_{p, q}=\frac{p^{\mu-1}-q^{\mu-1}}{p-q}$, and $\frac{[\mu-1]_{p, q}}{[\mu]_{p, q}}=$ $\frac{p^{\mu-1}-q^{\mu-1}}{p^{\mu}-q^{\mu}}=\frac{p^{\mu-1}}{p^{\mu}}\left(\frac{1-(q / p)^{\mu-1}}{1-(q / p)^{\mu}}\right)=\frac{1}{p}\left(\frac{1-(q / p)^{\mu-1}}{1-(q / p)^{\mu}}\right)$. For $0<\frac{q}{p}<1$, it is clear that $\lim _{\mu \rightarrow \infty}(q / p)^{\mu}=0$, $\lim _{\mu \rightarrow \infty}(q / p)^{\mu-1}=0$, and hence $\lim _{\mu \rightarrow \infty} \frac{[\mu-1]_{p, q}}{[\mu]_{p, q}}=\frac{1}{p}$.

Similarly other cases can be proved.

Case 2. When $0<q<1$ and $p=1$, then $\lim _{\mu \rightarrow \infty}[\mu]_{p, q}=\frac{1}{1-q}$ and $\lim _{\mu \rightarrow \infty} \frac{[\mu-1]_{p, q}}{[\mu]_{p, q}}=1$.

Case 3. When $0<p<q<1$, then $\lim _{\mu \rightarrow \infty}[\mu]_{p, q}=0$ and $\lim _{\mu \rightarrow \infty} \frac{[\mu-1]_{p, q}}{[\mu]_{p, q}}=\frac{1}{q}$.

Case 4. When $0<p<1$ and $q=1$, then $\lim _{\mu \rightarrow \infty}[\mu]_{p, q}=\frac{1}{1-p}$ and $\lim _{\mu \rightarrow \infty} \frac{[\mu-1]_{p, q}}{[\mu]_{p, q}}=1$.

Case 5. When $1 \leq q<p<\infty$, then $\lim _{\mu \rightarrow \infty}[\mu]_{p, q}=\infty$ and $\lim _{\mu \rightarrow \infty} \frac{[\mu-1]_{p, q}}{[\mu]_{p, q}}=\frac{1}{p}$.

Case 6. When $1 \leq p<q<\infty$, then $\lim _{\mu \rightarrow \infty}[\mu]_{p, q}=\infty$ and $\lim _{\mu \rightarrow \infty} \frac{[\mu-1]_{p, q}}{[\mu]_{p, q}}=\frac{1}{q}$.

Observe that in all the cases of choosing $p$ and $q \lim _{\mu \rightarrow \infty}[\mu]_{p, q} \neq \infty$ and $\lim _{\mu \rightarrow \infty} \frac{[\mu-1]_{p, q}}{[\mu]_{p, q}} \neq 1$ simultaneously. One can notice from the results for test functions $1, t$ and $t^{2}$ (especially the expression of $\left.L_{p, q}^{\mu, \alpha, \beta}\left(t^{2} ; x\right)\right)$ that in order to reach convergence result by the operator, we must have $\lim _{\mu \rightarrow \infty}[\mu]_{p, q}=\infty$ and $\lim _{\mu \rightarrow \infty} \frac{[\mu-1]_{p, q}}{[\mu]_{p, q}}=1$. When $p$ and $q$ are fixed numbers, then convergence can not be established. In order to get these limits, we need to choose sequences $p_{\mu}$ and $q_{\mu}$ based on following remarks.

Remark 3. Notice that in Case 1 and Case 3 of Remark (2) convergence can not be established for fixed $p$ and $q$. For the Case 2 and Case 6, we need a sequence $q=q_{\mu} \rightarrow 1$ and for Case 4 and Case 5, we need a sequence $p=p_{\mu} \rightarrow 1$.

Now we propose a theorem to overcome this shortcoming. Here one needs to choose sequences $p=p_{\mu}$ and $q=q_{\mu}$ in place of fixed $p$ and $q$ satisfying Theorem 1 . 
Theorem 1. Let $p=p_{\mu}$ and $q=q_{\mu}$ be the sequences of postive real numbers such that $\lim _{\mu \rightarrow \infty} p_{\mu}=1, \lim _{\mu \rightarrow \infty} q_{\mu}=1$ and $\lim _{\mu \rightarrow \infty} p_{\mu}^{\mu}=M_{1}, \lim _{\mu \rightarrow \infty} q_{\mu}^{\mu}=M_{2}$, where $M_{1}$ and $M_{2}$ are any positive real numbers. Then $\lim _{\mu \rightarrow \infty}[\mu]_{p, q}=\infty$ and $\lim _{\mu \rightarrow \infty} \frac{[\mu-1]_{p, q}}{[\mu]_{p, q}}=1$.

The above theorem has been widely used by many authors in different form for different operators based on $q$-integer and $(p, q)$-integer (see [8,9,34], [28, remark (5.1)]). Proof has not been provided yet, however in [16], Q.B. Cai and X.W. Xu has found the class of sequences $q_{\mu}$ and $p_{\mu}$ satisfying $0<q_{\mu}<p_{\mu} \leq 1$ and $q_{\mu} \rightarrow 1, p_{\mu} \rightarrow 1$ such that $\lim _{\mu \rightarrow \infty}[\mu]_{p_{\mu}, q_{\mu}}=\infty$. To prove the above Theorem 1, first we prove the following lemma.

Lemma 1. Let $a_{\mu}$ be a sequence of positive real numbers such that $\lim _{\mu \rightarrow \infty} a_{\mu}=1$ and $\lim _{\mu \rightarrow \infty}\left(a_{\mu}\right)^{\mu}=M$, where $M$ is any positive real number. Then $\lim _{\mu \rightarrow \infty}[\mu]_{a_{\mu}}=\infty$.

Proof. To prove this we need [13, Theorem 3.6.4 and Remark (a)].

Following cases are arised.

Case 1. $1<a_{\mu}<\infty$ for all $\mu \geq m_{0}$ and some $m_{0} \in \mathbb{N}$. We have $[\mu]_{a_{\mu}}=\frac{1-a_{\mu}{ }^{\mu}}{1-a_{\mu}}=$ $1+a_{\mu}+a_{\mu}{ }^{2}+\cdots+a_{\mu}{ }^{\mu-1}>1+1+1+\cdots+1=\mu$. Hence $\mu<[\mu]_{a_{\mu}}$ for all $\mu \geq m_{0}$. Thus $\lim _{\mu \rightarrow \infty}[\mu]_{a_{\mu}}=\infty$.

Case 2. $a_{\mu}=1$ for all $\mu \geq m_{0}$ and some $m_{0} \in \mathbb{N}$. We have $[\mu]_{a_{\mu}}=\mu$ for all $\mu \geq m_{0}$. Hence $\lim _{\mu \rightarrow \infty}[\mu]_{a_{\mu}}=\infty$.

Case 3. $0<a_{\mu}<1$ for all $\mu \geq m_{0}$ and some $m_{0} \in \mathbb{N}$. Let $b_{\mu}=\frac{1}{a_{\mu}}$. We have $[\mu]_{a_{\mu}}=\frac{1-a_{\mu}{ }^{\mu}}{1-a_{\mu}}=$ $\frac{\left(a_{\mu}\right)^{\mu}}{\left(a_{\mu}\right)}\left(\frac{\left(1 / a_{\mu}\right)^{\mu}-1}{\left(1 / a_{\mu}\right)-1}\right)=\frac{\left(a_{\mu}\right)^{\mu}}{\left(a_{\mu}\right)}[\mu]_{\frac{1}{a_{\mu}}}=\frac{\left(a_{\mu}\right)^{\mu}}{\left(a_{\mu}\right)}[\mu]_{b_{\mu}}$. Clearly $0<a_{\mu}<1 \Rightarrow 1<\frac{1}{a_{\mu}}<\infty \Rightarrow 1<b_{\mu}<\infty$ for all $\mu \geq m_{0}$. By Case $1 \lim _{\mu \rightarrow \infty}[\mu]_{b_{\mu}}=\infty$. So, $\lim _{\mu \rightarrow \infty}[\mu]_{a_{\mu}}=\frac{\lim _{\mu \rightarrow \infty}\left(a_{\mu}\right)^{\mu}}{\lim _{\mu \rightarrow \infty}\left(a_{\mu}\right)} \times \lim _{\mu \rightarrow \infty}[\mu]_{b_{\mu}}=\infty$.

Proof of Theorem 1. Let $a_{\mu}=\frac{p_{\mu}}{q_{\mu}}$,

$$
[\mu]_{p_{\mu}, q_{\mu}}=\frac{p_{\mu}^{\mu}-q_{\mu}^{\mu}}{p_{\mu}-q_{\mu}}=\frac{q_{\mu}^{\mu}}{q_{\mu}}\left(\frac{\left(\frac{p_{\mu}}{q_{\mu}}\right)^{\mu}-1}{\left(\frac{p_{\mu}}{q_{\mu}}\right)-1}\right)=\frac{q_{\mu}^{\mu}}{q_{\mu}}[\mu] \frac{p_{\mu}}{p_{\mu}}=\frac{q_{\mu}^{\mu}}{q_{\mu}}[\mu]_{a_{\mu}}, \quad \lim _{\mu \rightarrow \infty} a_{\mu}=\frac{\lim _{\mu \rightarrow \infty} p_{\mu}}{\lim _{\mu \rightarrow \infty} q_{\mu}}=1
$$

and $\lim _{\mu \rightarrow \infty}\left(a_{\mu}\right)^{\mu}=\frac{\lim _{\mu \rightarrow \infty}\left(p_{\mu}\right)^{\mu}}{\lim _{\mu \rightarrow \infty}\left(q_{\mu}\right)^{\mu}}=\frac{M_{1}}{M_{2}}>0$, hence by Lemma 1, we have $\lim _{\mu \rightarrow \infty}[\mu]_{a_{\mu}}=\infty$. Finally, $\lim _{\mu \rightarrow \infty}[\mu]_{p_{\mu}, q_{\mu}}=\frac{\lim _{\mu \rightarrow \infty}\left(q_{\mu}\right)^{\mu}}{\lim _{\mu \rightarrow \infty}\left(q_{\mu}\right)} \lim _{\mu \rightarrow \infty}[\mu]_{a_{\mu}}=\infty$.

Consider $\frac{[\mu-1]_{p_{\mu}, q_{\mu}}}{[\mu]_{p_{\mu}, q_{\mu}}}=\frac{p_{\mu}^{\mu-1}-q_{\mu}^{\mu-1}}{p_{\mu}^{\mu}-q_{\mu}^{\mu}}=\frac{q_{\mu}^{\mu-1}}{q_{\mu}^{\mu}}\left(\frac{\left(\frac{p_{\mu}}{q_{\mu}}\right)^{\mu-1}-1}{\left(\frac{p_{\mu}}{q_{\mu}}\right)^{\mu}-1}\right)=\frac{1}{q_{\mu}}\left(\frac{\left(a_{\mu}\right)^{\mu-1}-1}{\left(a_{\mu}\right)^{\mu}-1}\right)=\frac{1}{q_{\mu}}\left(\frac{1-\left(a_{\mu}\right)^{\mu-1}}{1-\left(a_{\mu}\right)^{\mu}}\right)$. Now, $\left(\frac{1-\left(a_{\mu}\right)^{\mu-1}}{1-\left(a_{\mu}\right)^{\mu}}\right)=\frac{\left(1-a_{\mu}\right)\left(1+a_{\mu}+a_{\mu}^{2}+\cdots+a_{\mu}^{\mu-2}\right)}{\left(1-a_{\mu}\right)\left(1+a_{\mu}+a_{\mu}^{2}+\cdots+a_{\mu}^{\mu-2}+a_{\mu}^{\mu-1}\right)}=\frac{1+a_{\mu}+a_{\mu}^{2}+\cdots+a_{\mu}^{\mu-2}+a_{\mu}^{\mu-1}-a_{\mu}^{\mu-1}}{1+a_{\mu}+a_{\mu}^{2}+\cdots+a_{\mu}^{\mu-2}+a_{\mu}^{\mu-1}}=\frac{[\mu]_{a_{\mu}}-a_{\mu}^{\mu-1}}{[\mu]_{a_{\mu}}}=$ $1-\frac{\left(a_{\mu}^{\mu}\right)}{a_{\mu}[\mu]_{a_{\mu}}}$. Hence $\frac{[\mu-1]_{p_{\mu, q}, q_{\mu}}}{[\mu]_{p_{\mu,} q_{\mu}}}=\frac{1}{q_{\mu}}\left(1-\frac{\left(a_{\mu}^{\mu}\right)}{a_{\mu}[\mu]_{a_{\mu}}}\right) \Rightarrow \lim _{\mu \rightarrow \infty} \frac{[\mu-1]_{p, q}}{[\mu]_{p, q}}=\lim _{\mu \rightarrow \infty} \frac{1}{q_{\mu}} \lim _{\mu \rightarrow \infty}\left(1-\frac{\left(a_{\mu}\right)^{\mu}}{a_{\mu}[\mu]_{a_{\mu}}}\right)=$ $1(1-0)=1$. 
Observe that Lemma 1 is quantum version of Theorem 1 . Now, we state a theorem which guarantees this approximation process to approximate every continuous function from the space $C[\alpha, \beta]$ by sequence of operators (6) for all $p>0$ and $q>0$, via Korovkin type approximation and using Remark 3.

Theorem 2. Let us consider sequences $0<p_{\mu}, q_{\mu}<\infty$ for each $\mu \in \mathbb{N}$ such that $\lim _{\mu \rightarrow \infty} p_{\mu}=1$, $\lim _{\mu \rightarrow \infty} q_{\mu}=1$ and $\lim _{\mu \rightarrow \infty} p_{\mu}^{\mu}=M_{1}, \lim _{\mu \rightarrow \infty} q_{\mu}^{\mu}=M_{2}$, where $M_{1}>0$ and $M_{2}>0$. Then for each $f \in C[\alpha, \beta], L_{p, q}^{\mu, \alpha, \beta}(f ; x)$ converges uniformly to $f$ on $C[\alpha, \beta]$.

Proof. Let us recall the following Korovkin's theorem.

Let $\left(T_{\mu}\right)$ be a sequence of positive linear operators from $C[\alpha, \beta]$ into $C[\alpha, \beta]$. Then $\lim _{\mu}\left\|T_{\mu}(f, x)-f(x)\right\|_{C[\alpha, \beta]}=0$, for all $f \in C[\alpha, \beta]$ if and only if $\lim _{\mu}\left\|T_{\mu}\left(f_{i}, x\right)-f_{i}(x)\right\|_{\mathcal{C}[\alpha, \beta]}=0$, for $i=0,1,2$, where $f_{0}(t)=1, f_{1}(t)=t$ and $f_{2}\left(t^{2}\right)=t^{2}$.

We need to show that if the sequence of operators converges for the test functions $1, t$ and $t^{2}$, then any continuous function can be approximated with the help of these positive linear operators.

Since $L_{p, q}^{\mu, \alpha, \beta}(f ; x)$ are positive linear operators, the Korovkin's theorem implies that

$$
L_{p, q}^{\mu, \alpha, \beta}(f ; x) \rightarrow f(x)
$$

if and only if $L_{p, q}^{\mu, \alpha, \beta}\left(t^{m}, x\right) \rightarrow x^{m}$ for all $x \in[\alpha, \beta]$ and $m=0,1,2$.

Following cases can arise.

Case 1. If we choose sequences $p_{\mu}$ and $q_{\mu}$ satisfying $0<q_{\mu}<p_{\mu} \leq 1$ for all $\mu \geq N_{0}$ and some $N_{0} \in \mathbb{N}$, such that $\lim _{\mu \rightarrow \infty} q_{\mu}=1, \lim _{\mu \rightarrow \infty} q_{\mu}^{\mu}=M, M>0$, then by squeeze theorem for sequences, we have $\lim _{\mu \rightarrow \infty} p_{\mu}=1$ and $\lim _{\mu \rightarrow \infty} q_{\mu}^{\mu} \leq \lim _{\mu \rightarrow \infty} p_{\mu}^{\mu} \leq 1$ and hence $\lim _{\mu \rightarrow \infty} p_{\mu}^{\mu}=K$, where $K$ is some positive real number.

Case 2. If we choose sequences $p_{\mu}$ and $q_{\mu}$ satisfying $0<p_{\mu}<q_{\mu} \leq 1$ for all $\mu \geq N_{0}$ and some $N_{0} \in \mathbb{N}$, such that $\lim _{\mu \rightarrow \infty} p_{\mu}=1, \lim _{\mu \rightarrow \infty} p_{\mu}^{\mu}=M, M>0$, then squeeze theorem for sequences implies that $\lim _{\mu \rightarrow \infty} q_{\mu}=1$ and $\lim _{\mu \rightarrow \infty} q_{\mu}^{\mu}=K$, where $K$ is some positive real number.

Case 3. When $1 \leq q_{\mu}<p_{\mu}<\infty$ for all $\mu \geq N_{0}$ and some $N_{0} \in \mathbb{N}$, such that $\lim _{\mu \rightarrow \infty} p_{\mu}=1$ and $\lim _{\mu \rightarrow \infty} p_{\mu}^{\mu}=M, M>0$, then by squeeze thorem we have $\lim _{\mu \rightarrow \infty} q_{\mu}=1$ and $\lim _{\mu \rightarrow \infty} q_{\mu}^{\mu}=K$, where $K$ is some positive real number.

Case 4. When $1 \leq p_{\mu}<q_{\mu}<\infty$ for all $\mu \geq N_{0}$ and some $N_{0} \in \mathbb{N}$, such that $\lim _{\mu \rightarrow \infty} q_{\mu}=1$ and $\lim _{\mu \rightarrow \infty} q_{\mu}^{\mu}=M, M>0$, then by squeeze thorem we have $\lim _{\mu \rightarrow \infty} p_{\mu}=1$ and $\lim _{\mu \rightarrow \infty} p_{\mu}^{\mu}=K$, where $K$ is some positive real number. implies

In all the cases we get $\lim _{\mu \rightarrow \infty}[\mu]_{p_{\mu}, q_{\mu}}=\infty, \lim _{\mu \rightarrow \infty} \frac{[\mu-1]_{p_{\mu}, q_{\mu}}}{[\mu]_{p_{\mu}, q_{\mu}}}=1$, and thus $\lim _{\mu \rightarrow \infty} \frac{p_{\mu}^{\mu-1} x}{[\mu]_{p_{\mu}, q_{\mu}}}=0$, which

$$
\begin{array}{r}
\alpha^{2}+\left(\frac{p_{\mu}^{\mu-1}}{[\mu]_{p_{\mu}, q_{\mu}}} \frac{(x-\alpha)}{(\beta-\alpha)}+\frac{q_{\mu}^{2}(x-\alpha)^{2}}{(\beta-\alpha)\left(p_{\mu}(\beta-x)+q_{\mu}(x-\alpha)\right)} \frac{[\mu-1]_{p_{\mu}, q_{\mu}}}{[\mu]_{p_{\mu}, q_{\mu}}}\right)(\beta-\alpha)^{2}+2 \alpha(x-\alpha) \\
\rightarrow \alpha^{2}+(x-\alpha)^{2}+2 \alpha(x-\alpha)=x^{2} .
\end{array}
$$

This completes the proof. 
Remark 4. To show the convergence of sequence of operators (6) to any continuous function defined on [0,1], authors of [28] have discussed only some particular part of Case 2, considered in the proof of Theorem 2. They have taken condition on choosing sequences $p_{\mu}$ and $q_{\mu}$ satisfying $0<q_{\mu}<p_{\mu} \leq 1$ such that $\lim _{\mu \rightarrow \infty} p_{\mu}=1, \lim _{\mu \rightarrow \infty} q_{\mu}=1$ and $\lim _{\mu \rightarrow \infty} p_{\mu}^{\mu}=1, \lim _{\mu \rightarrow \infty} q_{\mu}^{\mu}=1$, while we take more general condition on sequences $p_{\mu}$ and $q_{\mu}$ in above Theorem 2.

Theorem 3. Let $f \in C[\alpha, \beta], g(x)=f(\alpha+\beta-x)$. Then for any $p>0, q>0$,

$$
L_{p, q}^{\mu, \alpha, \beta}(f ; t)=L_{\frac{1}{p}, \frac{1}{q}}^{\mu, \alpha, \beta}(g ; \alpha+\beta-t) \quad \text { for } t \in[\alpha, \beta] .
$$

Proof. The proof of above theorem follows easily along the lines of [40] and using the following relations

$$
[\mu]_{p, q}=(p q)^{\mu-1}[\mu]_{\frac{1}{q}, \frac{1}{p}}, \quad \frac{p^{r}[\mu-r]_{p, q}}{[\mu]_{p, q}}=1-\frac{p^{r-\mu}[r]_{\frac{1}{q}, \frac{1}{p}}}{[\mu]_{\frac{1}{q}, \frac{1}{p}}}
$$

and

$$
\left[\begin{array}{l}
\mu \\
r
\end{array}\right]_{p, q}=\left[\begin{array}{l}
\mu \\
r
\end{array}\right]_{\frac{1}{q}, \frac{1}{p}} \frac{(p q)^{\frac{r(2 \mu-r+1)}{2}}}{(p q)^{\frac{r(r+1)}{2}}}
$$

Remark 5. For $p=1, \alpha=0$ and $\beta=1$ this equality coincides with [40, formula (10), Theorem 3].

\section{The Rate of Convergence}

In this section, rates of convergence of the operators (6) obtained by modulus of continuity and for the functions of Lipschitz type will be studied.

For any continuous function $f \in C[\alpha, \beta]$, the modulus of continuity $w(f ; \delta)$ is given by

$$
w(f ; \delta)=\sup _{u, x \in[\alpha, \beta],|x-u|<\delta}|f(x)-f(u)|
$$

and it satisfies the two conditions below

$$
\lim _{\delta \rightarrow 0} w(f ; \delta)=0 \quad \text { and } \quad|f(x)-f(u)| \leq w(f ; \delta)\left(\frac{|x-u|}{\delta}+1\right) \quad \text { for all } f \in E .
$$

Theorem 4. Let $p, q>0, \delta>0$ and $f \in C[a, b]$. We have

$$
\left\|L_{p, q}^{\mu, \alpha, \beta}(f ; x)-f(x)\right\|_{C[\alpha, \beta]} \leq 2 \omega\left(f ; \delta_{\mu}\right),
$$

where

$$
\delta_{\mu}=\left(\left(\frac{p^{\mu-1}(x-\alpha)}{[\mu]_{p, q}(\beta-\alpha)}+\frac{q^{2}(x-\alpha)^{2}}{(\beta-\alpha)(p(\beta-x)+q(x-\alpha))} \frac{[\mu-1]_{p, q}}{[\mu]_{p, q}}\right)(\beta-\alpha)^{2}-(x-\alpha)^{2}\right)^{\frac{1}{2}} .
$$

Proof. For $u \in[\alpha, \beta]$, we take

$$
\left|L_{p, q}^{\mu, \alpha, \beta}(f ; u)-f(u)\right| \leq w(f ; \delta)\left\{1+\frac{1}{\delta}\left(L_{p, q}^{\mu, \alpha, \beta}\left((x-u)^{2}: u\right)^{\frac{1}{2}}\right\}\right.
$$


Then using Lemma (2.2) we get

$$
\begin{aligned}
\left\|L_{p, q}^{\mu, \alpha, \beta}(f ; u)-f(u)\right\|_{C[\alpha, \beta]} \leq & w(f ; \delta)\left\{1+\frac{1}{\delta}\left(L_{p, q}^{\mu, \alpha, \beta}\left((x-u)^{2}: u\right)^{\frac{1}{2}}\right\}\right. \\
\leq & w(f ; \delta)\left\{1+\frac{1}{\delta}\left(\left(\frac{p^{\mu-1}(x-\alpha)}{[\mu]_{p, q}(\beta-\alpha)}\right.\right.\right. \\
& \left.\left.\left.+\frac{q^{2}(x-\alpha)^{2}}{(\beta-\alpha)(p(\beta-x)+q(x-\alpha))} \frac{[\mu-1]_{p, q}}{[\mu]_{p, q}}\right)(\beta-\alpha)^{2}-(x-\alpha)^{2}\right)^{\frac{1}{2}}\right\} .
\end{aligned}
$$

If we choose

$$
\delta_{\mu}=\left(\left(\frac{p^{\mu-1}(x-\alpha)}{[\mu]_{p, q}(\beta-\alpha)}+\frac{q^{2}(x-\alpha)^{2}}{(\beta-\alpha)(p(\beta-x)+q(x-\alpha))} \frac{[\mu-1]_{p, q}}{[\mu]_{p, q}}\right)(\beta-\alpha)^{2}-(x-\alpha)^{2}\right)^{\frac{1}{2}},
$$

then we have

$$
\left\|L_{p, q}^{\mu, \alpha, \beta}(f ; u)-f(u)\right\|_{C[\alpha, \beta]} \leq 2 \omega\left(f ; \delta_{\mu}\right) .
$$

So we have the desired result.

Now we compute the rate of convergence for the functions of Lipschitz class.

Let $f \in C[\alpha, \beta]$ and $0<\rho \leq 1$. We recall that $f \in \operatorname{Lip}_{M}(\rho)$ if $|f(u)-f(v)| \leq M|u-v|^{\rho}$ for all $u, v \in[\alpha, \beta]$.

Theorem 5. For all $f \in \operatorname{Lip}_{M}(\rho)$ we have $\left\|L_{p, q}^{\mu, \alpha, \beta}(f ; u)-f(u)\right\|_{C[\alpha, \beta]} \leq M \delta_{\mu}^{\rho}(u)$, where

$$
\delta_{\mu}=\left(\left(\frac{p^{\mu-1}(x-\alpha)}{[\mu]_{p, q}(\beta-\alpha)}+\frac{q^{2}(x-\alpha)^{2}}{(\beta-\alpha)(p(\beta-x)+q(x-\alpha))} \frac{[\mu-1]_{p, q}}{[\mu]_{p, q}}\right)(\beta-\alpha)^{2}-(x-\alpha)^{2}\right)^{\frac{1}{2}}
$$

and $M$ is a positive constant.

Proof. Let $f \in \operatorname{Lip} M(\rho)$ and $0<\rho \leq 1$. By (6) and linearity of $L_{r, p, q}^{\mu, \alpha, \beta}(f ; u)$, we have

$$
\left|L_{p, q}^{\mu, \alpha, \beta}(f ; u)-f(u)\right| \leq L_{p, q}^{\mu, \alpha, \beta}(|f(x)-f(u)|) \leq M L_{p, q}^{\mu, \alpha, \beta}\left(|x-u|^{\rho}\right) .
$$

Using the Hölder's inequality with $l=\frac{2}{\rho}$ and $r=\frac{2}{2-\rho}$, we have

$$
\left|L_{p, q}^{\mu, \alpha, \beta}(f ; u)-f(u)\right| \leq M\left(L_{p, q}^{\mu, \alpha, \beta}\left((x-u)^{2}\right)\right)^{\frac{\rho}{2}}
$$

if we choose $\delta=\delta_{\mu}$, then our proof is completed.

\section{Graphical Analysis}

Finally, let us consider the function $f(x)=2+\sin 5 x$ for graphical analysis. In Figures $1,2,3,4,5$ and 6 , we have presented approximation of this function by Lupaş post quantum Bernstein operators (6) on different intervals $[\alpha, \beta]$ for different values of $p, q$ and $\mu$.

Figures 1, 3 and 5 represent approximation of function $f(x)=2+\sin 5 x$ by Lupaş post quantum Bernstein operators (6) for $\mu=20$. Figures 2, 4 and 6 represent approximation of this function by Lupaş post quantum Bernstein operators (6) for $\mu=40$. If we compare a figure on 


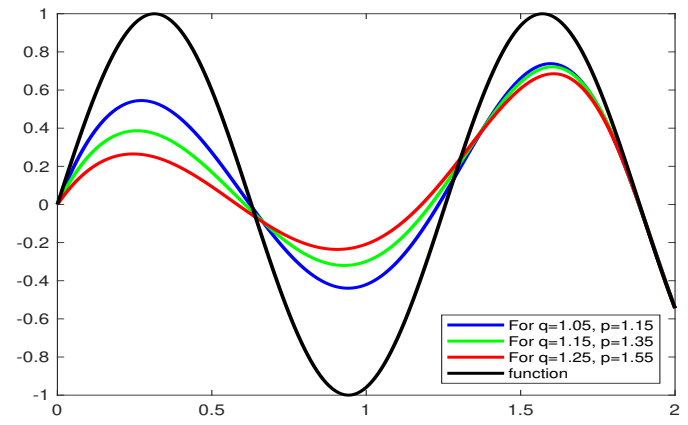

Figure 1: $\mu=20$

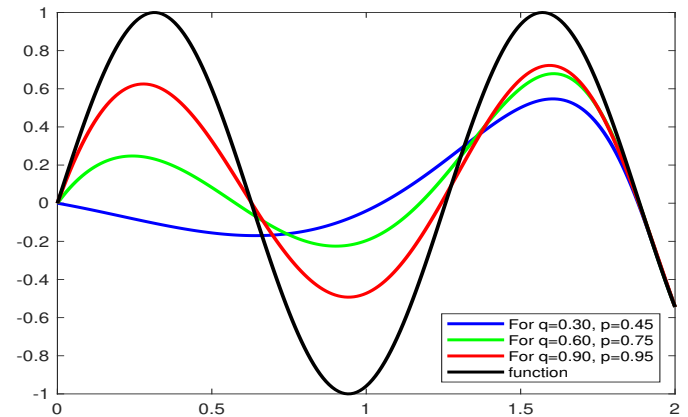

Figure 3: $\mu=20$

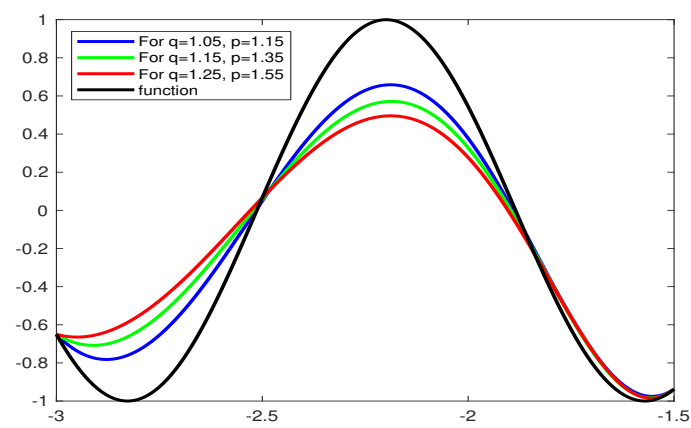

Figure 5: $\mu=20$

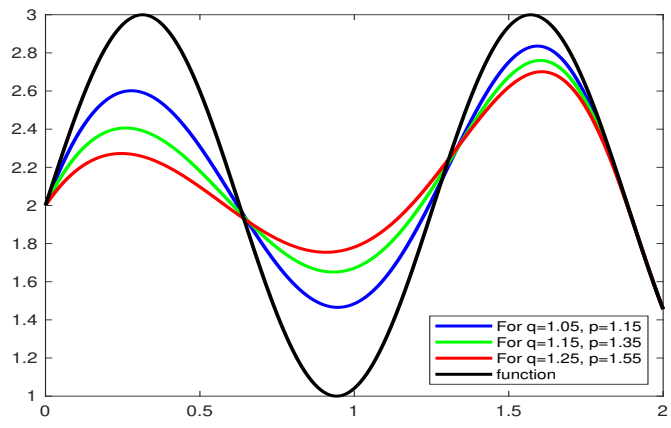

Figure 2: $\mu=40$

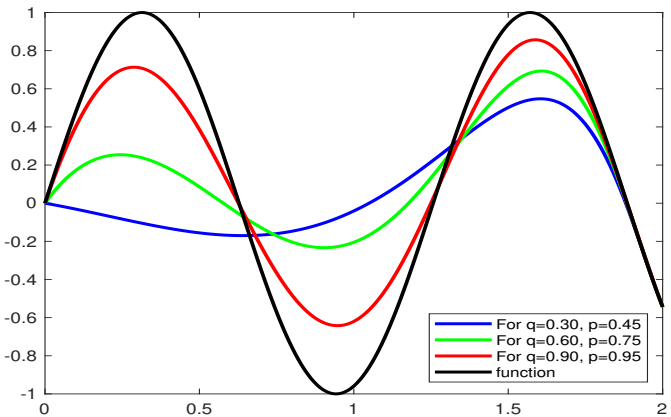

Figure 4: $\mu=40$

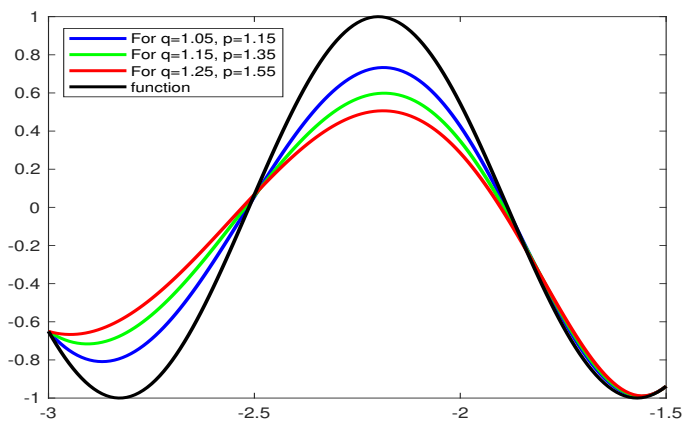

Figure 6: $\mu=40$

left side by a figure on right side, wich means that $\mu$ increases from 20 to 40, approximation becomes better for fixed $p$ and $q$, which is also theortically true by Theorem 2 .

Note, that Figures 1, 2 represent graphs on interval [0,2], and Figures 5 and 6 represent graphs on interval $[-3,-1.5]$, when $p$ and $q$ tends to 1 from right. The graphs of operators represented by blue colour are much closer to graph of function in comparison to graphs of operators represented by red and green colours. It is also theoretically true by Theorem 2 (Case 3 and Case 4), approximation becomes better, when sequences $p_{\mu}$ and $q_{\mu}$ converges to 1 from right. 


\section{References}

[1] Ali F.A.M., Karim S.A.A., Saaban A., Hasan M.K., Ghaffar A., Nisar K.S., Baleanu D. Construction of cubic timmer triangular patches and its application in scattered data interpolation. Mathematics 2020, 8 (2), 1-46. doi:10.3390/math8020159

[2] Ashraf P., Nawaz B., Baleanu D., Nisar K.S., Ghaffar A., Khan M.A.A., Akram S. Analysis of geometric properties of ternary four-point rational interpolating subdivision scheme. Mathematics 2020, 8 (3), 1-19. doi:10.3390/math8030338

[3] Acar T., Agrawal P.N., Kumar A.S. On a Modification of $(p, q)$-Szász-Mirakyan Operators. Complex Anal. Oper. Theory 2018, 12 (1), 155-167. doi:10.1007/s11785-016-0613-9

[4] Ilarslan H.G.I., Acar T. Approximation by bivariate ( $p, q)$-Baskakov-Kantorovich operators. Georgian Math. J. 2016, 25 (3), 397-407. doi:10.1515/gmj-2016-0057

[5] Acar T., Aral A., Mohiuddine S.A. Approximation by Bivariate $(p, q)$-Bernstein-Kantorovich Operators. Iran. J. Sci. Technol. Trans. A Sci. 2016, 42, 655-662. doi:10.1007/s40995-016-0045-4

[6] Sharma H., Maurya R., Gupta C. Approximation Properties of Kantorovich Type Modifications of $(p, q)$-MeyerKönig-Zeller Operators. Constr. Math. Anal. 2018, 1 (1), 58-72. doi:10.33205/cma.436071

[7] Mursaleen M., Nasiruzzaman M. Approximation of Modified Jakimovski-Leviatan-Beta Type Operators. Constr. Math. Anal. 2018, 1 (2), 88-98. doi:10.33205/cma.453284

[8] Acar T. $(p, q)$-Generalization of Szász-Mirakyan operators. Math. Methods Appl. Sci. 2016, 39 (10), $2685-2695$. doi:10.1002/mma.3721

[9] Acar T., Aral A., Mohiuddine S.A. On Kantorovich modification of $(p, q)$-Baskakov operators. J. Inequal. Appl. 2016, 2016 (98), 1-14. doi:10.1186/s13660-016-1045-9

[10] Acar T., Aral A., Mohiuddine S.A. On Kantorovich modification of $(p, q)$-Bernstein operators. Iran. J. Sci. Technol. Trans. A Sci. 2017, 42 (3), 1459-1464. doi:10.1007/s40995-017-0154-8

[11] Acar T., Aral A., Mursaleen M. Approximation by Baskakov-Durrmeyer operators based on $(p, q)$-integers. Math. Slovaca 2018, 68 (4), 897-906. doi:10.1515/ms-2017-0153

[12] Acar T., Mohiuddine S.A., Mursaleen M. Approximation by ( $p, q)$-Baskakov-Durrmeyer-Stancu operators. Complex Anal. Oper. Theory 2018, 12 (6), 1453-1468. doi:10.1007/s11785-016-0633-5

[13] Bartle R.G., Sherbert D.R. Introduction to Real Analysis. John Wiley and Sons, Inc., University of Illinois, Urbana-Champaign, 2010.

[14] Bernstein S.N. Démonstration du théorème de Weierstrass fondée sur le calcul des probabilités. Comm. Kharkov Math. Soc. 1912, 13, 1-2.

[15] Bèzier P.E. Numerical Control-Mathematics and applications. John Wiley and Sons, London, 1972.

[16] Cai Q.B., Xu X.W. A basic problem of $(p, q)$-Bernstein-type operators. J. Inequal. Appl. 2017, 140 (2017), 1-7. doi:10.1186/s13660-017-1413-0

[17] Cai Q.B., Cheng W.T. Convergence of $\lambda$-Bernstein operators based on $(p, q)$-integers. J. Inequal. Appl. 2020,35 (2020), 1-17. doi:10.1186/s13660-020-2309-y

[18] Goldman R. Pyramid Algorithms: A Dynamic Programming Approach to Curves and Surfaces for Geometric Modeling. The Morgan Kaufmann Series in Computer Graphics and Geometric Modeling. Elsevier Science, 2003.

[19] Farouki R.T., Rajan V.T. Algorithms for polynomials in Bernstein form. Comput. Aided Geom. Design 1988, 5 (1), 1-26. doi:10.1016/0167-8396(88)90016-7

[20] Han L., Chu Y., Qiu Z. Generalized Bèzier curves and surfaces based on Lupaş q-analogue of Bernstein operator. J. Comput. Appl. Math. 2014, 261, 352-363. doi:10.1016/j.cam.2013.11.016 
[21] Kadak U. On weighted statistical convergence based on $(p, q)$-integers and related approximation theorems for functions of two variables. J. Math. Anal. Appl. 2016, 443 (2), 752-764. doi:10.1016/J.JMAA.2016.05.062

[22] Kadak U., Mishra V.N., Pandey S. Chlodowsky type generalization of $(p, q)$-Szász operators involving Brenke type polynomials. Rev. R. Acad. Cienc. Exactas Fís. Nat. Ser. A Math. RACSAM 2018, 112 (4), 1443-1462.

[23] Kadak U., Mohiuddine S.A. Generalized statistically almost convergence based on the difference operator, which includes the $(p, q)$-Gamma function and related approximation theorems. Results Math. 2018, 73, article no. 9. doi:10.1007/s00025-018-0789-6

[24] Kadak U. Weighted statistical convergence based on generalized difference operator involving $(p, q)$-Gamma function and its applications to approximation theorems. J. Math. Anal. Appl. 2017, 448 (2), 1633-1650. doi: 10.1016/J.JMAA.2016.11.084

[25] Khan Kh., Lobiyal D.K., Kilicman A. A de Casteljau Algorithm for Bernstein type Polynomials based on $(p, q)$-integers. Appl. Appl. Math. 2018, 13 (2), 997-1017.

[26] Khan Kh., Lobiyal D.K., Kilicman A. Bézier curves and surfaces based on modified Bernstein polynomials. Azerb. J. Math. 2019, 9 (1), 3-21.

[27] Khan Kh. Generalized Bézier curves and their applications in computer aided geometric design. Ph.D. Thesis, SC \& SS, JNU New Delhi, 2019.

[28] Khan Kh., Lobiyal D.K. Bézier curves based on Lupaş $(p, q)$-analogue of Bernstein function in CAGD. Jour. Comput. Appl. Math. 2017, 317, 458-477.

[29] Karim S.A.A., Saaban A., Skala V., Ghaffar A., Nisar K.S., Baleanu D. Construction of new cubic Bézierlike triangular patches with application in scattered data interpolation. Adv. Differ. Equ. 2020, 151 (2020). doi:10.1186/s13662-020-02598-w

[30] Lupaş A. A q-analogue of the Bernstein operator. Semin. Numer. Stat. Calculus 1987, 9, 85-92.

[31] Korovkin P.P. Linear operators and approximation theory. Hindustan Publishing Corporation, Delhi, 1960.

[32] Lewanowicz S., Woźny P. Generalized Bernstein polynomials. BIT 2004, 44 (1), 63-78. doi:10.1023/B:BITN.0000025086.89121.d8

[33] Mishra V.N., Patel P.On generalized integral Bernstein operators based on q-integers. Appl. Math. Comput. 2014, 242 (1), 931-944. doi:10.1016/j.amc.2014.05.134

[34] Mohiuddine S.A., Alotaibi A., Acar T. Durrmeyer type $(p, q)$-Baskakov operators preserving linear functions. J. Math. Inequal. 2018, 12 (4), 961-73. doi:10.7153/jmi-2018-12-73

[35] Mursaleen M., Ansari K.J., Khan A. On ( $p, q)$-analogue of Bernstein Operators. Appl. Math. Comput. 2015, 266, 874-882.

[36] Mursaleen M., Ansari K.J., Khan A. Some approximation results by (p,q)-analogue of Bernstein-Stancu operators. Appl. Math. Comput. 2015, 264, 392-402.

[37] Mursaleen M., Nasiruzzaman Md., Nurgali A. Some approximation results on Bernstein-Schurer operators defined by $(p, q)$-integers. J. Inequal. Appl. 2015, 249. doi:10.1186/s13660-015-0767-4

[38] Mursaleen M., Nasiruzzaman Md., Ansari K. J., Alotaibi A. Generalized ( $p, q)$-Bleimann-Butzer-Hahn operators and some approximation results. J. Inequal. Appl. 2017, 310. doi:10.1186/s13660-017-1582-x

[39] Mursaleen M., Nasiruzzaman Md., Khan A., Ansari K.J. Some approximation results on Bleimann-Butzer-Hahn operators defined by $(p, q)$-integers. Filomat 2016, 30 (3), 639-648.

[40] Ostrovska S. On the Lupaş q-analogue of the Bernstein operator. Rocky Mountain J. Math. 2006, 36 (5), $1615-1629$. doi:10.1216/rmjm/1181069386

[41] Phillips G.M. A generalization of the Bernstein polynomials based on the q-integers. ANZIAM J. 2000, 42 (1), 79-86. doi:10.1017/S1446181100011615

[42] Phillips G.M. Bernstein polynomials based on the q-integers. Annals Numer. Math. 1997, 4, 511-518. 
[43] Phillips G.M. Interpolation and Approximation by Polynomials. Springer, New York, 2003.

[44] Rababah A., Manna S. Iterative process for G2-multi degree reduction of Bézier curves. Appl. Math. Comput. 2011, 217 (20), 8126-8133. doi:10.1016/j.amc.2011.03.016

[45] Simeonova P., Zafirisa V., Goldman R. q-Blossoming: A new approach to algorithms and identities for q-Bernstein bases and q-Bézier curves. J. Approx. Theory 2012, 164 (1), 77-104. doi:10.1016/j.jat.2011.09.006

[46] Rao N., Wafi A. (p,q)-Bivariate-Bernstein-Chlodowsky operators. Filomat 2018, 32 (2), 369-378. doi:10.2298/FIL1802369R

[47] Rao N., Wafi A. Bivariate-Schurer-Stancu operators based on ( $p, q)$-integers. Filomat 2018, 32 (4), $1251-1258$. doi:10.2298/FIL1804251R

[48] Zulkifli N.A.B., Karim S.A.A., Shafie A.B., Sarfraz M., Ghaffar A., Nisar K.S. Image interpolation using a rational bi-cubic Ball. Mathematics 2019, 7 (11), 1045. doi:10.3390/math7111045

Received 27.11.2020

Revised 12.01.2020

Хан А., Ільяс М., Мансурі М.С., Мурсалін М. Постквантові за Аупашом оператори Бернштейна над довільними компактними інтервалами // Карпатські матем. публ. - 2021. — Т.13, №3. — С. 734-749.

У цій статті розглянуто постквантові за Аупашом оператори Бернштейна над довільним замкнутим і обмеженим інтервалом, побудованими за допомогою Аупашаівських постквантових базисів Бернштейна. Завдяки властивості, що ці базиси $є$ інваріантними відносно масштабування та відносно трансляцій, отримані результати на довільних інтервалах є важливими з точки зору обчислень. Аосліджено апроксимаційні властивості постквантових за операторів Бернштейна на довільних компактних інтервалах на основі теореми типу Коровкіна. Обговорено більш загальну ситуацію для всіх можливих випадків шодо збіжності послідовності постквантових за Аупашом операторів до будь-якої неперервної функції, визначеної на компактному інтервалі. Обчислено швидкість збіжності за модулем неперервності та функціями класу Аіпшица. Аля демонстрації апроксимації неперервних функцій постквантовими за Аупашом операторами Бернштейна на різних компактних інтервалах представлено графічний аналіз за допомогою програми MATLAB.

Ключові слова і фрази: постквантове числення, постквантовий базис Бернштейна, постквантовий оператор Бернштейна, модуль неперервності, критерій збіжності, швидкість збіжності. 